\title{
Label-free imaging immune cells and collagen in atherosclerosis with two-photon and second harmonic generation microscopy
}

\author{
Chunqiang Li ${ }^{*}$, , Riikka K. Pastila ${ }^{\dagger}, *$ and Charles P. Lin ${ }^{\dagger}$ \\ *Department of Physics \\ The University of Texas at El Paso \\ El Paso, Texas 79968, USA \\ ${ }^{\dagger}$ Wellman Center for Photomedicine \\ and Center for Systems Biology \\ Massachusetts General Hospital \\ Harvard Medical School \\ 185 Cambridge St., Boston \\ Massachusetts 02114, USA \\ ${ }^{\star} S T U K$ - Radiation and Nuclear Safety Authority \\ Laippatie 4, FI-00880 Helsinki, Finland \\ §cli@utep.edu
}

Received 4 October 2015

Accepted 16 December 2015

Published 30 January 2016

\begin{abstract}
Atherosclerosis has been recognized as a chronic inflammation disease, in which many types of cells participate in this process, including lymphocytes, macrophages, dendritic cells (DCs), mast cells, vascular smooth muscle cells (SMCs). Developments in imaging technology provide the capability to observe cellular and tissue components and their interactions. The knowledge of the functions of immune cells and their interactions with other cell and tissue components will facilitate our discovery of biomarkers in atherosclerosis and prediction of the risk factor of rupture-prone plaques. Nonlinear optical microscopy based on two-photon excited autofluorescence and second harmonic generation (SHG) were developed to image mast cells, SMCs and collagen in plaque ex vivo using endogenous optical signals. Mast cells were imaged with two-photon tryptophan autofluorescence, SMCs were imaged with two-photon NADH autofluorescence, and collagen were imaged with SHG. This development paves the way for further study of mast cell degranulation, and the effects of mast cell derived mediators such as induced synthesis and activation of matrix metalloproteinases (MMPs) which participate in the degradation of collagen.
\end{abstract}

Keywords: Two-photon microscopy; mast cells; atherosclerosis.

$\S_{\text {Corresponding author. }}$

This is an Open Access article published by World Scientific Publishing Company. It is distributed under the terms of the Creative Commons Attribution 4.0 (CC-BY) License. Further distribution of this work is permitted, provided the original work is properly cited. 


\section{Introduction}

Vascular diseases, e.g., coronary heart disease and stroke, are the leading cause of morbidity and mortality in United States and expected to become in the whole world by 2020. Atherosclerosis, the formation of lipid-rich plaques in arteries is the major underlying process leading to vascular diseases. Although low-density lipoprotein (LDL), which damages endothelial cells and smooth muscle cells (SMCs) and induces inflammation, is the major risk factor in the initiation of the atherosclerosis, our knowledge has drastically changed since last decade, as the role of inflammation and immune response in atherosclerosis is prominent as demonstrated in many observations. ${ }^{1,2}$ Presently atherosclerosis has been recognized as a chronic inflammatory disease. ${ }^{3}$ Inflammation plays important roles in initiation, atheroma progression and complication and has become a therapeutic target in atherosclerosis and inflammatory markers become gauges of atherosclerotic risk. ${ }^{3,4}$ The immune cells involved in atherogenesis, atheroma progression and complication include macrophages, lymphocytes, neutrophils and mast cells. ${ }^{5}$ Therefore, understanding the biological process and finding effective therapeutic targets in atherosclerosis require the knowledge of immune cells functions mentioned above and their interactions with other cells and tissue components. Theses knowledge will facilitate our discovery of biomarkers in atherosclerosis and prediction of the risk factor of rupture-prone plaques. Among the immune cells involved in atherosclerosis, monocytes and later converted macrophages and $\mathrm{T}$ lymphocytes in plaque have been studied intensively. Recent discoveries have pointed to mast cells as another critical cellular component in atherosclerosis due to their critical roles in collagen degradation and regulating SMCs. ${ }^{6}$ Mast cell derived proteases and cytokines play important roles in lipid metabolism, vascular wall inflammation, plaque calcification and destabilization. ${ }^{7-9}$ It is shown that there is significant positive correlation between density of mast cell chymase and tryptase and disease progression. ${ }^{10}$ Mast cells promote atherosclerosis by releasing proinflammatory cytokines interleukin-6 (IL-6) and interferon- $\gamma$ (INF$\gamma) .{ }^{11}$ In vitro, the proteases released by mast cells induce synthesis and activation of matrix metalloproteinases (MMPs), which participate in the degradation of collagen. ${ }^{12,13}$ Collagen degradation leads to atherosclerotic complication such as plaque rupture. Smooth muscle cells change from a contractile to a proliferative phenotype, which synthesizes collagen matrix, during the progress of plaque. The survival and well-being of SMCs is critical for plaque stability. Experiments on cultured SMCs had shown that activated mast cells could induce apoptosis, inhibit proliferation and collagen expression of SMCs through mast cell-derived tumor necrosis factor- $\alpha$ (TNF- $\alpha)$, transforming growth factor- $\beta$ (TGF- $\beta$ ), heparin and chymase. ${ }^{14}$ To further delineate the role of mast cells in atherosclerotic plaque formation and progression in vivo, it is necessary to visualize mast cells and their activation and interaction with the collagen matrix and SMCs.

By sensing targeted molecules, molecular imaging provides information of fundamental biological processes and cells. Magnetic resonance imaging (MRI), computed tomography (CT), single-photonemission CT (SPECT) and near infrared fluorescence imaging, have been applied on vascular disease research. ${ }^{15}$ These imaging modalities not only enable the assessment of the vascular wall morphology but also the component of the wall and bring our observation ability into the cellular and molecular level. Most of the imaging experiments require exogenous contrast agents, e.g., magnetic nanoparticles, radioactive positron emitters or protease-activatable agents. Utilizing endogenous signals will overcome the obstacle of contrast agent development, reduce the possible interference with intrinsic local environment and make the applications on in vivo human study possible.

Optical imaging modalities, e.g., optical coherence tomography (OCT), have imaged macrophage and foam cells distribution in plaque. ${ }^{16}$ But the contrast in OCT images comes from the reflection of the light from the tissue, which lacks molecular information. Two-photon microscopy (TPM) has shown enormous potential for the imaging of dynamic molecular and cellular level behaviors in intact tissue, and it can provide information that would be indiscernible in population measurements and completely absent in vitro. Immunologists have adopted this technique to study leukocyte activities and interactions, such as the motility and trafficking of lymphocytes, and lymphocytes' interactions with dendritic cells (DCs) following immune response. ${ }^{17}$ The application of TPM on atherosclerosis research is still in its primitive stage. Collagen and elastin have been imaged with second harmonic generation (SHG) and TPM in vivo without 
exogenous labeling. ${ }^{18}$ Although leukocytes had been imaged with TPM in atheroma, the fluorophores in these experiments are green fluorescence protein (GFP) in leukocytes in transgenic mice or labeled fluorescent antibodies. ${ }^{18,19}$

Recent results demonstrate that mast cells can be imaged in vivo in mouse tissue with two-photon excited endogenous tryptophan fluorescence. ${ }^{20} \mathrm{In}$ addition, collagen can be imaged with SHG microscopy $^{21,22}$ in atheroma and SMCs can be imaged with NADH fluorescence from TPM. In this paper, we demonstrated imaging these three cellular and extracellular components in atheroma with these nonlinear optical microscopy techniques.

\section{Materials and Methods}

\subsection{Two-photon microscope}

The imaging platform is a home-built two-photon microscope. The light source for performing two-photon excitation on tryptophan is a $590 \mathrm{~nm}$ femtosecond laser system, i.e., a femtosecond Titanium/sapphire laser (Spectra-Physics Maitai HP, $750 \mathrm{~nm}, 2.5 \mathrm{~W}, 100 \mathrm{fs}$ ) pumps an optical parametric oscillator (OPO, Spectra-Physics, OPAL, $1180 \mathrm{~nm}$, $300 \mathrm{~mW}, 200 \mathrm{fs}$ ) and the output from the OPO was converted to $590 \mathrm{~nm}$ with $70 \mathrm{~mW}$ power through SHG inside a beta barium borate (BBO) crystal. The fluorescence spectrum of tryptophan centers around $350 \mathrm{~nm}$. NADH is excited with the Titani$\mathrm{um} /$ sapphire laser output at $750 \mathrm{~nm}$ and the fluorescence signal is detected at $400-460 \mathrm{~nm}$. Collagen is imaged with the Titanium/sapphire output at $750 \mathrm{~nm}$ and the SHG signal is detected at $375 \mathrm{~nm}$. The laser beam was reflected to a fast spinning polygonal mirror (Lincoln Laser, 480 revolutions/s) and a fast swing galvanometer-mounted mirror (Cambridge Technology, $30 \mathrm{~Hz}$ ) to obtain the twodimensional (2D) raster scan on sample. A diachronic beam splitter (Semrock, FF660-FDi01) is used to separate the excitation and emission beam; an Olympus water immersion microscope objective lens with a $60 \times$, NA1.2 is used to focus the laser beam on the sample. The excitation wavelength can be tuned by the Maitai HP control programmer on computer, and the excitation power on the sample can be adjusted by combining a quarter wave plate and a linear polarizer as a laser attenuator. The UV detection channel has two band pass filters (Semrock, FF01-357/44) to detect tryptophan autofluorescence. The blue detection channel has two band pass filters (Semrock, FF01-450/60) to detect NADH fluorescence. While performing SHG imaging the filters in the UV detection channel is changed to another two bandpass filters (Semrock, FF01-377/50). Fluorescence signals in three channel are detected by a photomultiplier tube (PMT) (Hamamatsu R3896), respectively. The PMT outputs are acquired by a frame grabber (Matrox Solios $\mathrm{eA} / \mathrm{XA}$ ) and recorded on personal computer at 30 frames/s. Reference 23 presents details of this microscope setup.

\subsection{Cell culture}

Mast cells (MC/9, ATCC CRL-8306) were purchased from ATCC for in vitro imaging. MC/9 cell line is a cloned mast cell line derived from the fetal liver of a (B6 XA/J)F1 mouse. Cell culture protocol was provided by the supplier.

\subsection{Mouse models}

For animal imaging experiments, BALB/c, C57BL/ 6 , mast cell deficient $\mathrm{B} 6 \mathrm{~W} / \mathrm{W}^{V}$ (WBB6F1/J-Kit ${ }^{\mathrm{W}} /$ $\mathrm{Kit}^{\mathrm{W}-\mathrm{v}}$, Jackson Laboratory), and atherosclerosisprone ApoE knockout mice (Jackson Laboratory) were imaged on ear pinna following administration of ketamine $(100 \mathrm{mg} / \mathrm{Kg})$ and xylazine $(15 \mathrm{mg} / \mathrm{Kg})$ anesthesia mixture. These mice were placed in a temperature controlled tube and the ear skin was flattened on a glass slide using Methocel gel $2 \%$. Inflammation was induced by UVB illumination in the BALB/c mouse ear pinna.

\section{Results}

\subsection{In vivo mast cell imaging in mouse skin}

Using tryptophan autofluorescence, we observed vessel-like structures in the dermis of BALB/c mice with dermal cells nearby Fig. 1(a). The vasculature was confirmed by observation of leukocytes flow in vivo and by colocalization of GFP signal in a Tie2 GFP mouse whose endothelial cells express the green fluorescent protein. ${ }^{24}$ In Fig. 1(a) we also observed bright dermal cells residing close to the vasculature (white arrow). It is known that skin mast cells reside close to vasculature. These dermal cells had a size of about $20 \mu \mathrm{m}$ diameter, which fits 


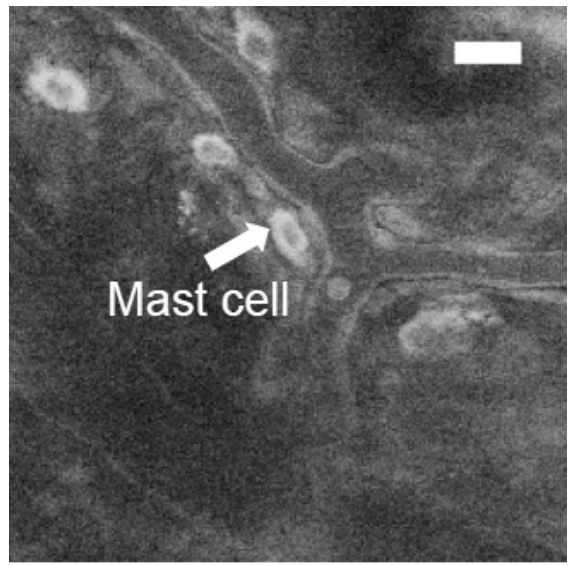

(a)

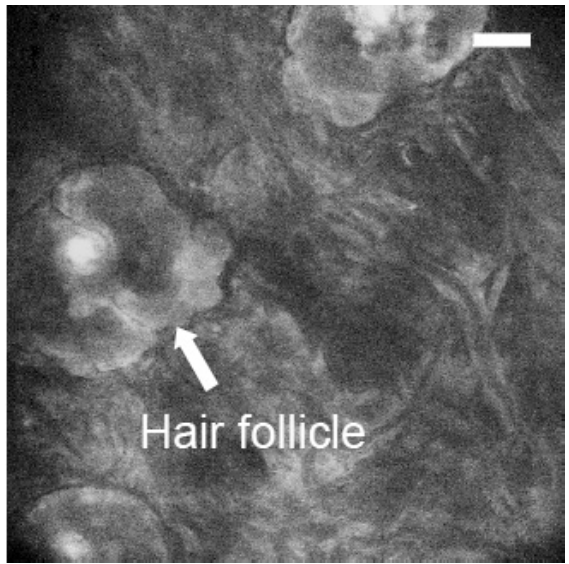

(b)

Fig. 1. Mast cells imaging with tryptophan autofluorescence (a) dermal mast cells in BALB/c mice reside close to blood vasculature, (b) mast cells are not present in mast cell-deficient mice $\left(\mathrm{B} 6 \mathrm{~W} / \mathrm{W}^{V}\right)$ dermis. (Bar: $20 \mu \mathrm{m}$ in (a), and $30 \mu \mathrm{m}$ in (b)).

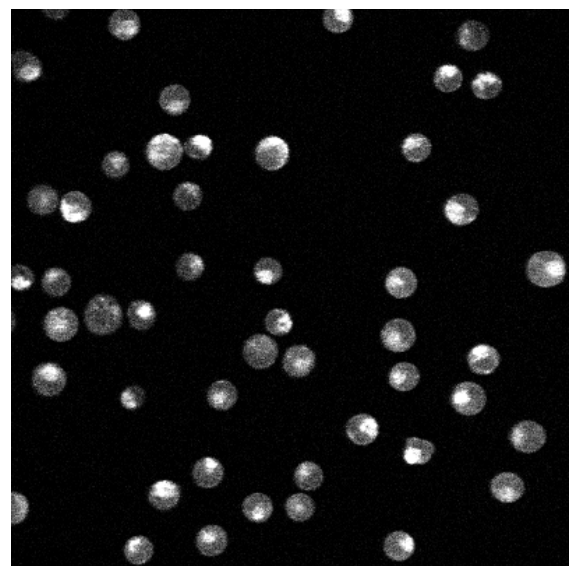

(a)

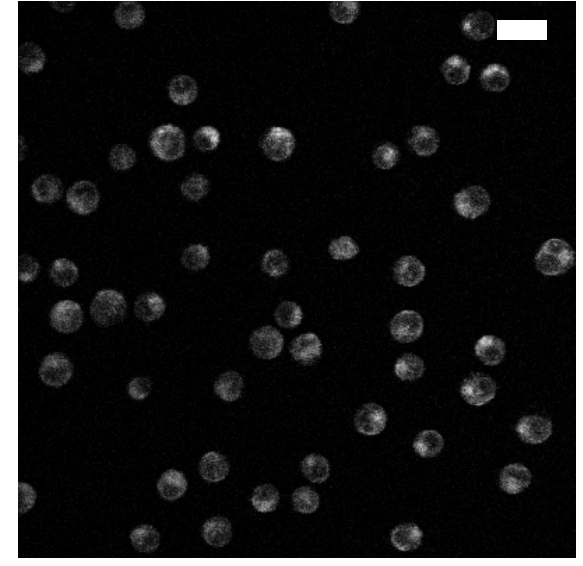

(b)

Fig. 2. In vitro mast cell degranulation process (a) image right after adding compound 48/80 and, (b) 20 min post adding compound 48/80. (Bar: $20 \mu \mathrm{m}$ ).

mast cell size. ${ }^{20}$ We have also imaged $\mathrm{B} 6 \mathrm{~W} / \mathrm{W}^{V}$ mice (WBB6F1/J-Kit ${ }^{\mathrm{W}} / \mathrm{Kit}^{\mathrm{W}-\mathrm{v}}{ }^{\text {, Jackson Labora- }}$ tory), in which mast cells are deficient, as shown in Fig. 1(b). There was no dermal cells similar to those in BALB/c mouse. This observation suggested the possibility of imaging mast cells with tryptophan autofluorescence using two-photon excitation. Imaging mast cell engraftment in mast cell deficient mice further confirmed that these dermal cells were mast cells. ${ }^{20}$

\subsection{Imaging mast cell degranulation}

The in vitro mast cell degranulation process was studied with time-lapse tryptophan autofluorescence imaging. Mast cells (MC/9, ATCC) in culture media (Fig. 2(a)) right after adding compound 48/80, a mast cell stimulator $(200 \mu \mathrm{l}, 10 \mu \mathrm{g} / \mathrm{ml})$, were imaged with tryptophan autofluorescence. All the mast cells in the field of view released their granules within $20 \mathrm{~min}$ and show less intensity in fluorescence imaging (Fig. 2(b)). This degranulation process had a burst mode where all granules in a single cell were secreted simultaneously. ${ }^{20}$ This burst happened at different time points for different cells. The granule size of mast cells varied between different residing tissue types and the typical diameter was $0.3-1 \mu \mathrm{m}$. Our current twophoton microscope had a lateral resolution about $250 \mathrm{~nm}$, which is capable of imaging individual granules during this degranulation process. 


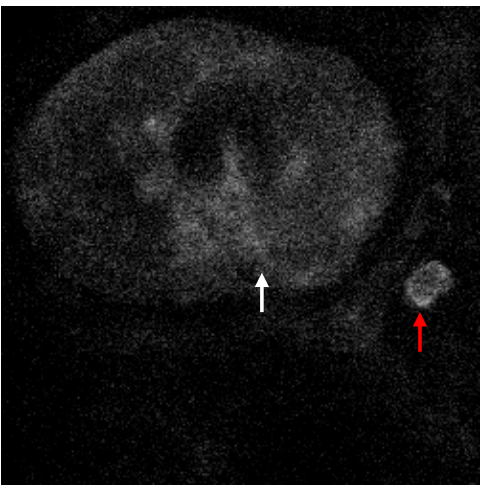

(a)

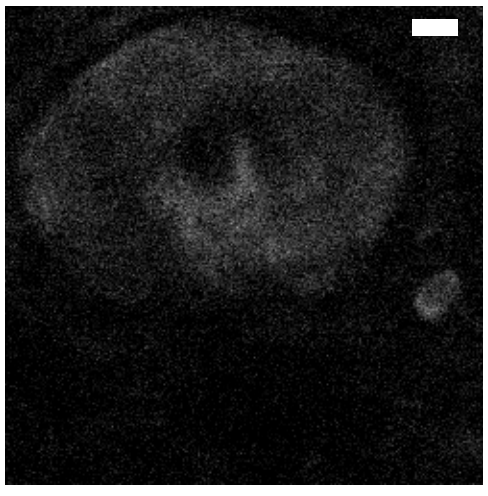

(b)

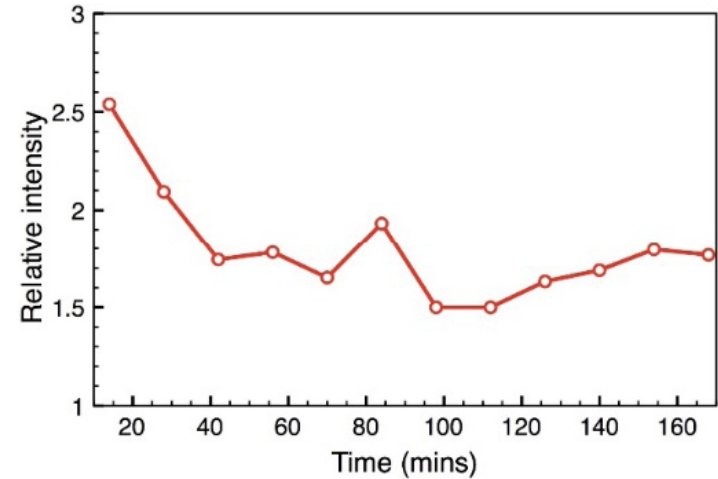

(c)

Fig. 3. In vivo mast cell degranulation process (a) image right 14 min post UVB illumination, (b) 100 min post UVB illumination and (c) individual granule fluorescence intensity count. (Bar: $20 \mu \mathrm{m}$ ).

UVB (290-320 nm) illumination on skin can induce inflammation depending on the dose. And it has been reported that mast cells degranulate to induce inflammation during this process. ${ }^{25}$ We have used this model to induce inflammation on C57BL6 mouse ear skin and observed mast cells degranulation in vivo. The UVB dose was $5 \mathrm{~kJ} / \mathrm{m}^{2}$. Two of the time series images over $3 \mathrm{~h}$ are shown in Fig. 3. To quantify the mast cell granule fluorescence intensity change, an area of 40 pixels with high intensity in the mast cell (red arrow) was chosen and the mean intensity of these pixels were calculated. To compensate the fluorescence change due to artifacts such as optical alignment drifting, a baseline was defined as the mean intensity of another structure with detectable autofluorescence signal, in this case a sebaceous gland nearby (white arrow). The relative intensity is the mast cells intensity divided by the baseline intensity. The relative intensity had a quick drop between 20 and $40 \mathrm{~min}$ and remains relatively flat afterward (Fig. 3(c)). The degranulation process of mast cells after UVB illumination under similar dose measured by immunohistochemical staining had been conducted before and this quick release of granules happened within $1 \mathrm{~h}$ after illumination. ${ }^{25}$ Our observation provides a new method of monitoring mast cell degranulation process in vivo during immunological responses.

\subsection{Mast cell ex vivo imaging in plaque}

In plaque mast cells mainly reside in the shoulder region. To investigate the feasibility of applying tryptophan autofluorescence on imaging mast cells in plaque, we have imaged the lesions from atherosclerosis-prone ApoE knockout mice descending thoracic aorta (black box by the heart in Fig. 4(a)) ex vivo. This mouse had been on Western diet for

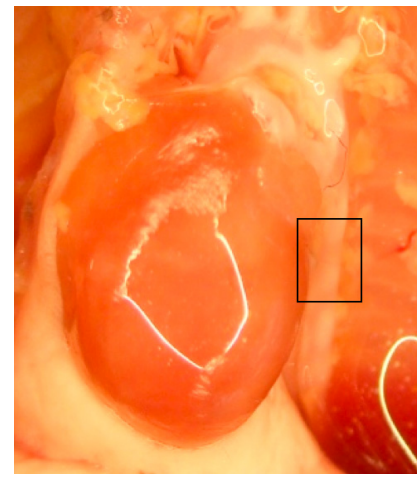

(a)

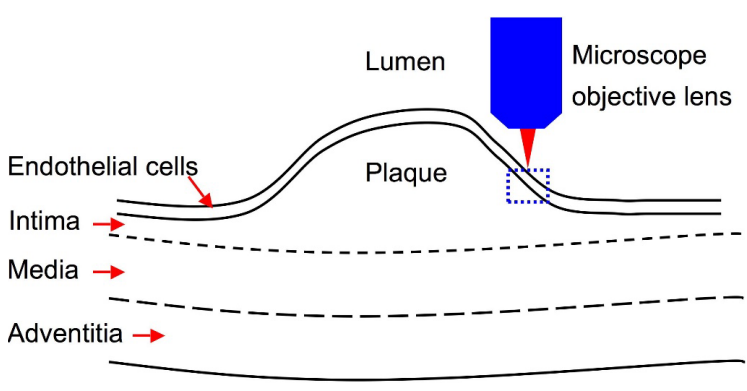

(b)

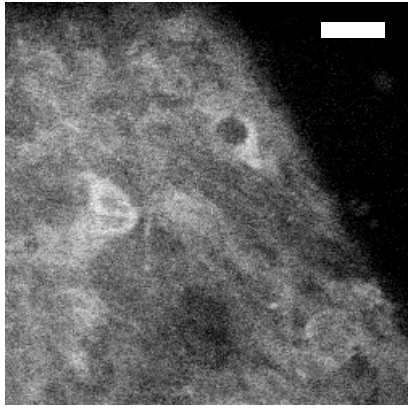

(c)

Fig. 4. Ex vivo mast cell imaging in atherosclerotic lesion (a) heart and aorta photo, (b) schematic drawing of the imaging setup and (c) mast cells in the shoulder region. (Bar: $20 \mu \mathrm{m}$ ). 
26 weeks to develop atherosclerosis before it was sacrificed. The aorta piece was dissected so that it can be imaged from the lumen side. The schematic imaging setup is shown in Fig. 4(b). As shown in Fig. 4(c) there were two discernable cellular components in the shoulder region (blue arrows). The major immunological cellular components in plaque include macrophages, monocytes, foam cells, $\mathrm{T}$ cells and mast cells. It has been reported that such immune cells has strong tryptophan autofluorescence. ${ }^{24}$ Further experiments, such as immunohistological staining, are needed to confirm the cell type in this image.

\subsection{Ex vivo smooth muscle cell imaging in plaque}

Muscle cells have been commonly imaged with twophoton NADH fluorescence for the study of metabolic status. ${ }^{26}$ We imaged muscles cells in vivo on mouse ear skin with NADH fluorescence as shown in Fig. 5(a). This image reveals unmistakable A-Z lines of muscle cells. We had also imaged the smooth muscles cells in normal aorta and plaque ex vivo with the same setup. The images are shown in Figs. 5(b) and 5(c), respectively for normal and diseased artery. In the media layer of a normal aorta, SMCs arranged in a well-aligned pattern and had high NADH fluorescence intensity (Fig. 5(b)). The SMCs in plaque (white arrow in Fig. 5(c)) had much lower NADH fluorescence than the normal SMCs. Although this phenomenon may be due to degraded fluorescence signal from tissue scattering in plaque. Further quantification of NADH fluorescence can be achieved by imaging FAD fluorescence simultaneously and taking the ratio between $\mathrm{NADH}$ and FAD intensity.

\subsection{Ex vivo collagen imaging in atheroma}

Collagen is the main protein of connective tissue in animals. In skin, collagen makes up the extracellular matrix. Second harmonic generation microscopy is an established method for imaging collagen in vivo and quantifying their structural change. The amino acid content in collagen measured by the ionexchange chromatographic method has shown no tryptophan in human bone and tendon collagen, therefore it will induce cross talk to tryptophan autofluorescence signal. In our experiment collagen in normal aorta wall and plaque had been imaged with SHG microscopy ex vivo. The collagen SHG image in intima is shown in Fig. 6(a). In the intima layer collagen was the connective tissue with wellaligned orientation (Fig. 6(a)). In the adventitia layer, collagen was much denser with wavy structure as shown in Fig. 6(b). The morphology of collagen in plaque (Fig. 6(c)) was quite different from the normal collagen in artery. As collagen is responsible for the stability of plaque, this morphology can be used to diagnose the instability of plaque. The collagen fiber length and curvature can be calculated from three-dimensional (3D) images for further quantitative analysis.

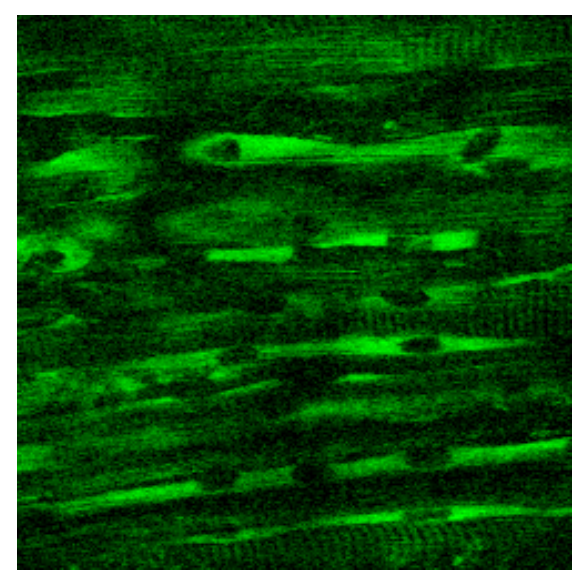

(a)

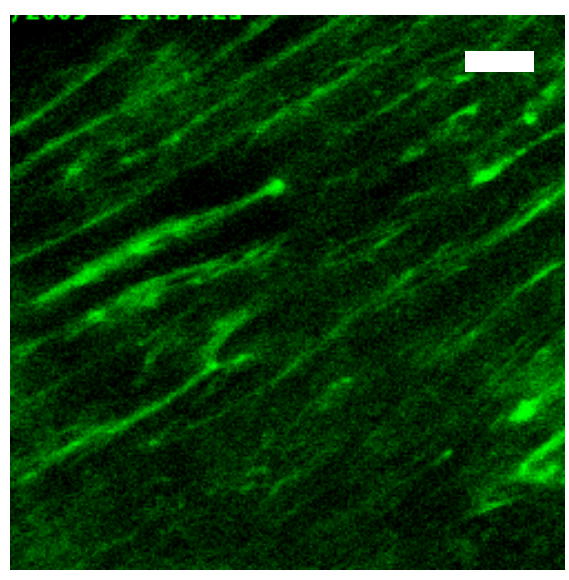

(b)

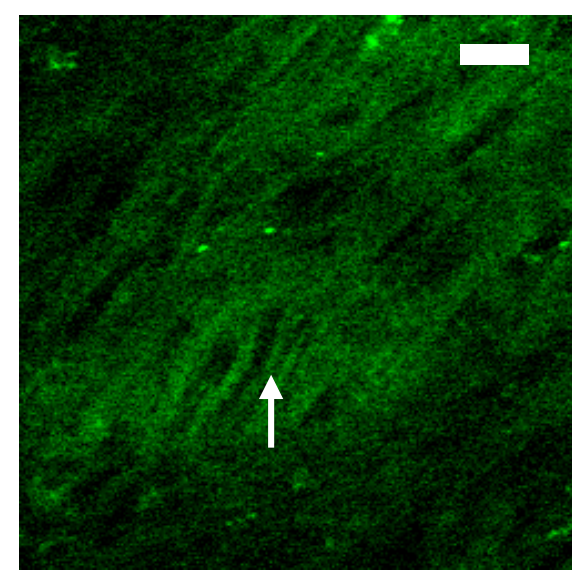

(c)

Fig. 5. (a) In vivo muscle cells imaging in mouse ear skin (b) ex vivo smooth muscles cells in normal aorta and (c) ex vivo smooth muscle cells in plaque. (Bar: $20 \mu \mathrm{m}$ in (a), $30 \mu \mathrm{m}$ in (b) and (c)). 


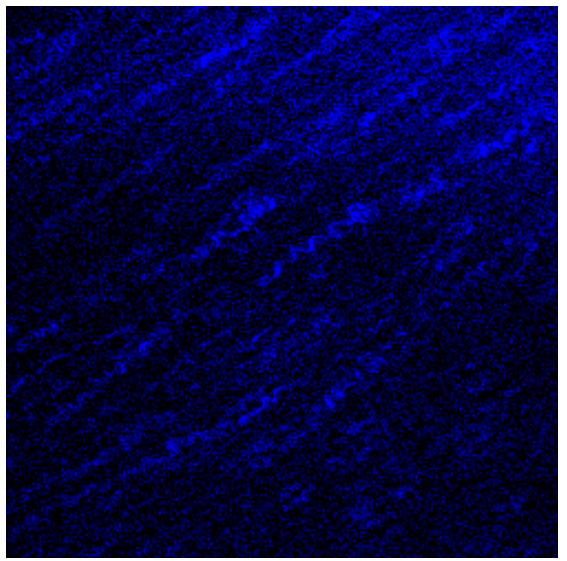

(a)

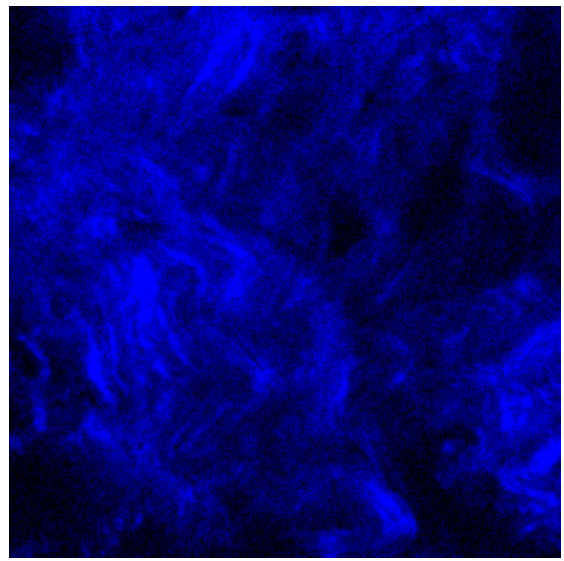

(b)

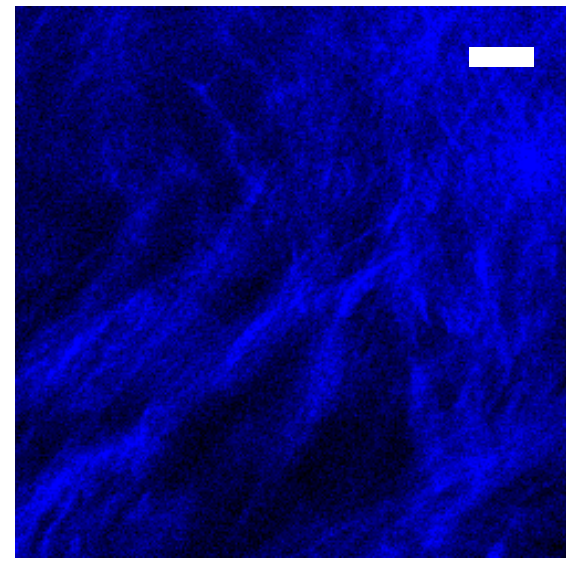

(c)

Fig. 6. Ex vivo SHG imaging of collagen in (a) intima, (b) adventitia and (c) plaque. (Bar: $20 \mu \mathrm{m})$.

\section{Discussion}

In this study, we have demonstrated using a twophoton and SHG microscopy to image mast cells, SMCs and collagen in atherosclerosis plaque ex vivo without exogenous labeling. Our result has shown that mast cells have strong tryptophan autofluorescence, SMCs can be imaged with NADH autofluorescence, and collagen can be imaged with SHG signal. These results could lead to further development on imaging these cellular and tissue components, such as image mast cell in atherosclerotic lesion in vivo and follow their degranulation process; correlate collagen morphological change in vivo in SHG images with mast cell activities; and image SMCs in vivo and measure their metabolic status using endogenous NADH/FAD fluorescence. These future experiments could provide evidence that how these three components play important roles in atherosclerosis and reveal their interactions in vivo.

The challenge of translating these nonlinear optical imaging modalities into live animal imaging is the optical accessibility to atheroma lesions from lumen. Fiber based micro-optical coherence tomography ( $\mu \mathrm{OCT})$ has demonstrated the ability to image subcellular structure of human coronary atherosclerosis. ${ }^{27}$ Therefore, it is promising to develop minimally invasive fiber-optic based nonlinear optical micro-endoscopy (TPM and SHG) to characterize atherosclerotic plaques in live animals to study mast cell accumulation, activation, collagen degradation, and SMCs metabolic status.

\section{Acknowledgement}

This study was supported by United States NIH P41 EB015903-02S1 grant awarded to CPL. We would like to thank Drs. Crystal M. Ripplinger and Farouc A. Jaffer for their help on the mouse model of atherosclerotic plaque.

\section{References}

1. R. Ross, "Athresclerosis - an inflammatory disease," N. Engl. J. Med. 340(2), 115-126 (1999).

2. A. J. Lusis, "Atherosclerosis," Nature 407(6801), 233-241 (2000).

3. P. Libby, "Inflammation in atherosclerosis," Nature 420(6917), 868-874 (2002).

4. G. K. Hansson, P. Libby, "The immune response in atherosclerosis: A double-edged sword," Nat. Rev. Immunol. 6(7), 508-519 (2006).

5. C. Weber, A. Zernecke, P. Libby, "The multifaceted contributions of leukocyte subsets to atherosclerosis: Lessons from mouse models," Nat. Rev. Immunol. 8(10), 802-815 (2008).

6. P. Libby, G.-P. Shi, "Mast cells as mediators and modulators of atherogenesis," Circulation 115(19), 2471-2473 (2007).

7. P. T. Kovanen, "Mast cells: Multipotent local effector cells in atherothrombosis," Immun. Rev. 217(1), 105-122 (2007).

8. I. Bot, G.-P. Shi, P. T. Kovanen, "Mast cells as effectors in atherosclerosis," Arterioscler. Thromb. Vascu. Biol. 35(2), 265-271 (2015).

9. G.-P. Shi, I. Bot, P. T. Kovanen, "Mast cells in human and experimental cardiometabolic diseases," Nat. Rev. Cardiol. 12(11), 643-658 (2015). 
10. L. S. Ramalho et al., "Role of mast cell chymase and tryptase in the progression of atherosclerosis: Study in 44 autopsied cases," Ann. Diagn. Pathol. 17(1), 28-31 (2013).

11. J. Sun et al., "Mast cells promote atherosclerosis by releasing proinflammatory cytokines," Nat. Med. 13(6), 719-724 (2007).

12. P. T. Kovanen, "Mast cells and degradation of pericellular and extracellular matrices: Potential contributions to erosion, rupture and intraplaque haemorrhage of atherosclerotic plaques," Biochem. Soc. Trans. 035(5), 857-861 (2007).

13. J. L. Johnson et al., "Activation of matrix-degrading metalloproteinases by mast cell proteases in atherosclerotic plaques," Arterioscler. Thromb. Vasc. Biol. 18(11), 1707-1715 (1998).

14. M. J. Leskinen, P. T. Kovanen, K. A. Lindstedt, "Regulation of smooth muscle cell growth, function and death in vitro by activated mast cells - a potential mechanism for the weakening and rupture of atherosclerotic plaques," Biochem. Pharmacol. 66(8), 1493-1498 (2003).

15. F. A. Jaffer, P. Libby, R. Weissleder, "Molecular imaging of cardiovascular disease," Circulation 116 (9), 1052-1061 (2007).

16. J. Oh et al., "Detection of macrophages in atherosclerotic tissue using magnetic nanoparticles and differential phase optical coherence tomography," J. Biomed. Opt. 13(5), 054006-054008 (2008).

17. R. N. Germain et al., "Dynamic imaging of the immune system: Progress, pitfalls and promise," Nat. Rev. Immunol. 6(7), 497-507 (2006).

18. W. Yu et al., "In vivo imaging of atherosclerotic plaques in apolipoprotein $\mathrm{E}$ deficient mice using nonlinear microscopy," J. Biomed. Opt. 12(5), 054008-054010 (2007).
19. R. T. A. Megens et al., "Two-photon microscopy on vital carotid arteries: Imaging the relationship between collagen and inflammatory cells in atherosclerotic plaques," J. Biomed. Opt. 13(4), 044022-10 (2008).

20. C. Li, R. K. Pastila, C. P. Lin, "Imaging immune response of skin mast cells in vivo with two-photon microscopy," SPIE Photonics West, San Francisco, CA (2012).

21. A. Zoumi, A. Yeh, B. J. Tromberg, "Imaging cells and extracellular matrix in vivo by using second-harmonic generation and two-photon excited fluorescnece," Proc. Natl. Acad. Sci. USA 99(17), 11014 (2007).

22. P. J. Campagnola, L. M. Loew, "Second-harmonic imaging microscopy for visualizing biomolecular arrays in cells, tissues and organisms," Nat. Biotech. 21(11), 1356-1360 (2003).

23. I. Veilleux et al., "In vivo cell tracking with video rate multimodality laser scanning microscopy," IEEE J. Sel. Top. Quant. Electron. 14(1), 10-18 (2008).

24. C. Li et al., "Imaging leukocyte trafficking in vivo with two-photon-excited endogenous tryptophan fluorescence," Opt. Express 18(2), 988-999 (2010).

25. L. J. Walsh, "Ultraviolet B irradiation of skin induces mast cell degranulation and release of tumour necrosis factor-[alpha]," Immunol. Cell. Biol. 73(3), 226-233 (1995).

26. S. Huang, A. A. Heikal, W. W. Webb, "Two-photon fluorescence spectroscopy and microscopy of NAD $(\mathrm{P}) \mathrm{H}$ and flavoprotein," Biophys. J. 82(5), 2811-2825 (2002).

27. L. Liu et al., "Imaging the subcellular structure of human coronary atherosclerosis using micro-optical coherence tomography," Nat. Med. 17(8), 10101014 (2011). 